\title{
Application of time-frequency analysis to transient data from centrifuge earthquake testing
}

\author{
David E. Newland and Gary D. Butler* \\ Department of Engineering, University of Cambridge, \\ Trumpington Street, Cambridge, CB2 1PZ, UK \\ E-mail:den@eng.cam.ac.uk,butlerg@wes.army.mil
}

Received 7 July 1999

Revised 9 March 2000

Centrifuge model experiments have generated complex transient vibration data. New algorithms for time-frequency analysis using harmonic wavelets provide a good method of analyzing these data. We describe how the experimental data have been collected and show typical time-frequency maps obtained by the harmonic wavelet algorithm. Some preliminary comments on the interpretation of these maps are given in terms of the physics of the underlying model. Important features of the motion that are not otherwise apparent emerge from the analysis. Later papers will deal with their more detailed interpretation and their implications for centrifuge modeling.

\section{Fourier spectra}

The technique of analysing a time-varying signal by filtering out its frequency components is widely used. Data in digital form is filtered by the fast Fourier transform algorithm to produce a set of coefficients which describe the amplitude and phase of the signal's harmonic components. Intrinsic to the analysis is the concept that the recorded signal is one period of a regularly repeating, periodic signal. Each (complex) coefficient describes the amplitude and phase of one harmonic. Each coefficient is a property of the complete signal analysed: for each harmonic there is one amplitude and one phase.

This works well when the signal is stationary (or approximately so) in the statistical sense. In other words,

*Also at US Army Engineer Research and Development Center. when its statistical properties are unchanging with time. For transient signals, that is not the case. For example, the natural frequency of a pendulum depends on the amplitude of oscillation. Large amplitudes have a longer period than small amplitudes. If a pendulum is started from a large amplitude and allowed to swing unchecked, its period will progressively decrease with time. Its harmonic coefficients therefore change with time. For such a transient signal, Fourier analysis produces a spectra of harmonic coefficients which describe the average frequency properties of the signal and do not show the changing frequency content with time.

There are three main methods of time-frequency analysis which attempt to show how the frequency content of a signal changes with time. These are: (1) the short-time Fourier transform (STFT), which generates a spectrogram (also called a sonogram), (2) the Wigner-Ville method of generating time-frequency distributions and (3) the harmonic wavelet method of constructing wavelet maps. Important features of the spectrogram are discussed in the paper by Hodges et al. [3], Wigner-Ville frequency distributions in Mark [5] and the book by Cohen [2], and the theory of wavelet maps in the first author's book and papers listed [8]. A comparison of the three methods is given in Newland [14].

All three methods generate a (real) function of time and frequency which can be plotted to generate a surface on the time-frequency plane. This function is a measure of energy (or mean-square). The time and frequency scales are both divided into small intervals, and the energy function describes how energy is shared over time and frequency. Specifically, for each combination of a particular frequency interval (bandwidth) and time increment, the energy function specifies how much energy lies in that combination.

The usual method of displaying this surface is by a contour map in two dimensions and the practical requirement is to plot a map with high definition to show, as accurately as possible, the changing frequen- 
cy pattern of the measured signal. Both the STFT and Wigner-Ville methods are constant bandwidth methods. In contrast, the harmonic wavelet method allows the bandwidth of adjacent frequency terms to be chosen arbitrarily. Because the wavelet transform acts as a variable $Q$ filter, where $Q$ is the ratio of center frequency to bandwidth, it has greater flexibility than the other two methods. For this reason, the authors have found that the harmonic wavelet method provides a good practical method for analysing transient vibration records. It is applied in this paper to the analysis of records obtained from analysing the large-amplitude motion of soil samples to transient excitation. These are interesting because the response properties of the soil change with time and are dependent on frequency and amplitude. The changing time-frequency behavior would be completely excluded by traditional Fourier analysis.

\section{Features of time-frequency maps}

Time-frequency maps are two-dimensional contour diagrams of a three-dimensional surface. The choice of contour levels and of colored shading (if used) can make substantial differences to the way a diagram appears.

Usually the map's axes are time (plotted horizontally) and frequency (vertically). Time is defined as the center of the time window (the mean time of the wavelet). Frequency is the center of the frequency window (the mean frequency of the wavelet). Only wavelets with a narrow frequency band are effective for time-frequency analysis, otherwise good frequency resolution is impossible. Subject to this narrow-band proviso, wavelets of any kind may be used, but harmonic wavelets are particularly suitable because their spectrum is confined to a compact, defined frequency band [7].

An important restriction on all time-frequency analysis is that precise frequency information can only be obtained if there is a long enough record. The total energy of a signal is broken down into parts, with each part associated with a particular combination of time and frequency intervals. These intervals must be finite. If the bandwidth is small, the time interval is large, and vice-versa. Only for an infinitely-long record can the bandwidth approach zero, because, if $\mathrm{B}$ is the bandwidth in $\mathrm{Hz}$ and $\mathrm{T}$ the time interval in seconds then it can be shown that the following inequality must be satisfied:

$$
B T \geqslant 1
$$

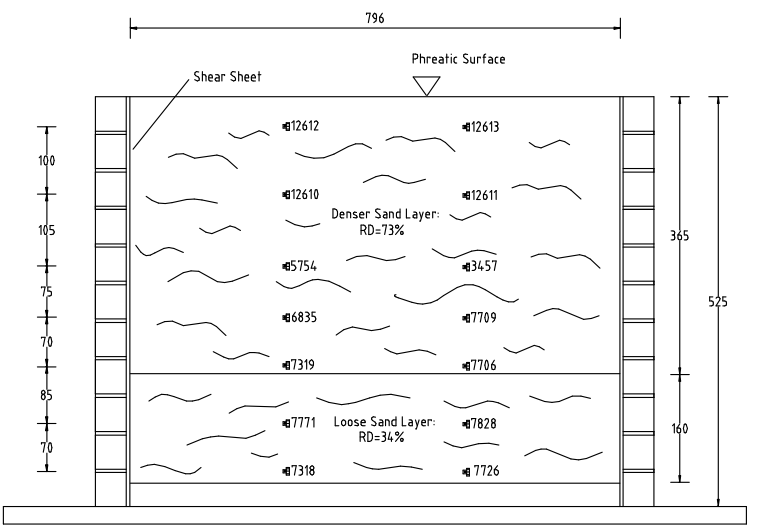

Fig. 1. Instrumentation layout.

Strictly, this is true for a stationary signal of bandwidth $B$ and duration $T$, but it serves also as an approximate guide in the general case of a transient signal. Although the method of analysis may use a grid of frequency and time intervals smaller than specified by (1), each value of the calculated energy function gives a result averaged over an area of the time-frequency plane which must be at least the minimum area defined by (1).

\section{Experimental system}

Tests were conducted onboard the US Army Centrifuge at the Waterways Experiment Station (WES) in Vicksburg, Mississippi. Excitation to the base of the model is supplied using the WES stored angular momentum actuator (SAM). The experimental system comprises a saturated soil model poured at two relative densities within a flexible-laminar container. The container functions as an equivalent shear beam whose shear modulus was designed to match that of the enclosed soil medium. At the bottom of the model, sand was poured at a density $1576 \mathrm{~kg} / \mathrm{m}^{3}$ for $160 \mathrm{~mm}$ and $1670 \mathrm{~kg} / \mathrm{m}^{3}$ for the remaining $365 \mathrm{~mm}$ to the top of the container. As seen in Figure 1, accelerometers are stacked vertically within the soil with four in the bottom layer and ten in the top layer.

Transducers used for the experiment were miniature piezo-electric accelerometers manufactured by D.J. Birchall of Cheltenham, England. Each transducer is $10 \mathrm{~mm}$ in height and has a mass of approximately $5 \mathrm{gms}$ (Fig. 2). Tests conducted previously at WES show that these accelerometers have an accuracy of $5 \%$ within the frequency range of $20 \mathrm{~Hz}$ to $2 \mathrm{kHz}$. Their natural frequency when embedded in sand is estimated to be 


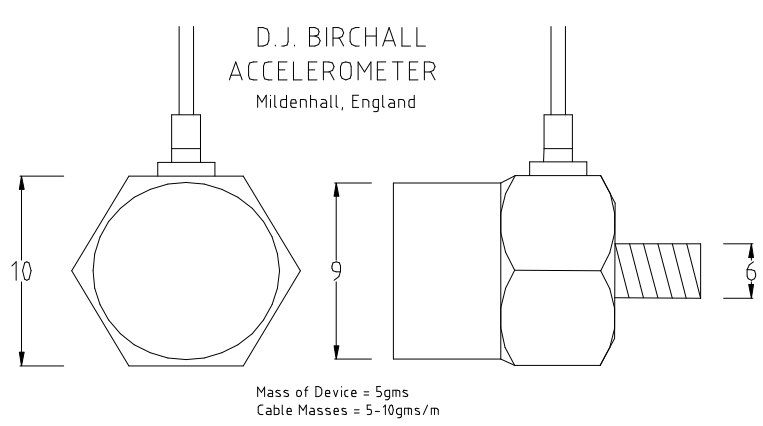

Fig. 2. Miniature piezo-electric accelerometer (all dimensions in $\mathrm{mm})$.

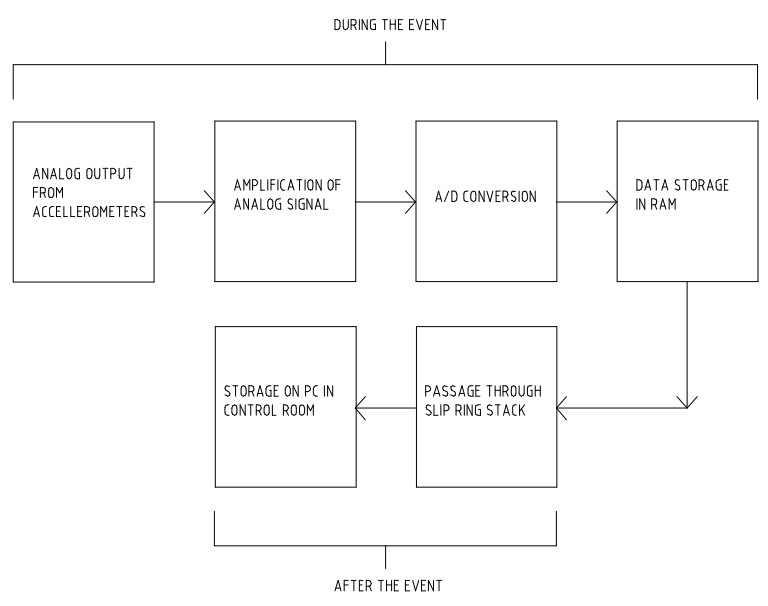

Fig. 3. Diagram of data acquisition time sequence.

about $4 \mathrm{kHz}$ compared with frequencies of interest up to about $400 \mathrm{~Hz}$.

Each transducer was carefully oriented in the soil to record the resulting horizontal motion (horizontal direction in Fig. 1 within the saturated model. To prevent the seepage of pore fluid into the transducers, each instrument was sealed using silicon rubber.

The cables from the accelerometers in the model are connected into a 32 channel junction box which contains the signal amplifiers. Data is retrieved from the junction box during flight using an on-board control data acquisition system (CDAQS). The CDAQS was developed in Cambridge and uses both a high speed A/D converter and Tattletale ${ }^{\circledR}$ data logger. The data acquisition system is activated in the centrifuge control room using a solid state relay timing device. After the shaking event, the data is uploaded from the CDAQS unit through a stacked slip ring set to the centrifuge control room where it is stored on a PC for analysis (Fig. 3).

Results reported in this paper were acquired from a model subjected to $50 \mathrm{~g}$ of normal acceleration from
TYPICAL CENTRIFUGE RESULTS
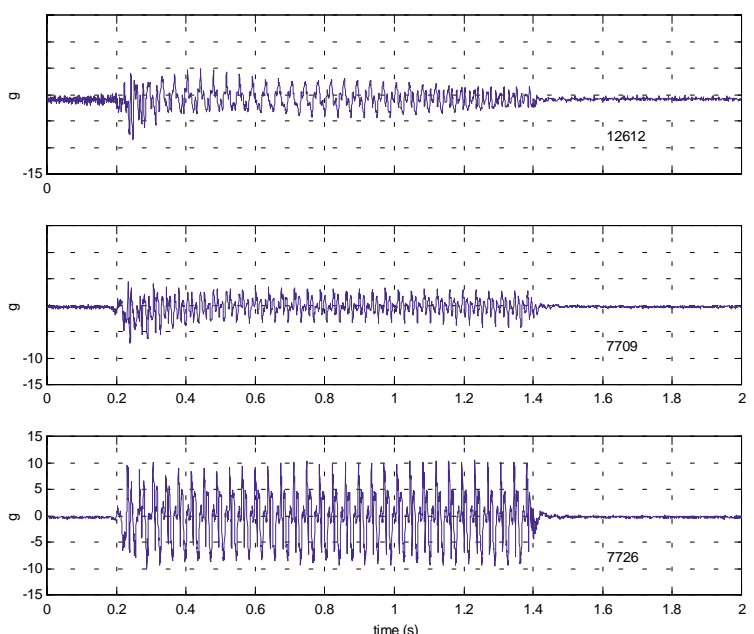

Fig. 4. Recorded accelerations (units of g) in the test model: measured in dense sand near the top of the container, in dense sand at the middle and at the bottom of the container in an underlying looser sand layer.

the centrifuge (vertically in Fig. 1). The input motion to the base of the model container had a fundamental frequency of $27 \mathrm{~Hz}$ with a displacement amplitude of $1.49 \mathrm{~mm}$. The duration of the shake was set to $1.2 \mathrm{sec}-$ onds. For purely harmonic movement, these displacements correspond to a lateral acceleration amplitude of approximately $4.4 \mathrm{~g}$.

The SAM actuator uses the stored energy from a set of rotating flywheels as a means of delivering shaking energy to a centrifuge earthquake model. Because of the complexity of the mechanism, a purely harmonic motion of the base of the model cannot be achieved and the significant harmonics present in Fig. 5 indicate the non-harmonic character of the motion obtained. By using an eccentric and a high speed hydraulic clutch, the system has the ability to quickly deliver the stored energy as an oscillatory motion to the base of the model container.

The operation of the system begins by driving the flywheels to the desired input frequency before engaging the drive train and the model container by activation of the hydraulic clutch. During the initial engagement period, the mechanical system undergoes a large shock due to the rapid and immense transfer of energy. This initial excitation is followed by an input of oscillatory energy for a specified duration. After this time, the clutch is disengaged therefore de-coupling the flywheels' motion from that of the model container. At the point of disengagement, a second jolt is produced 

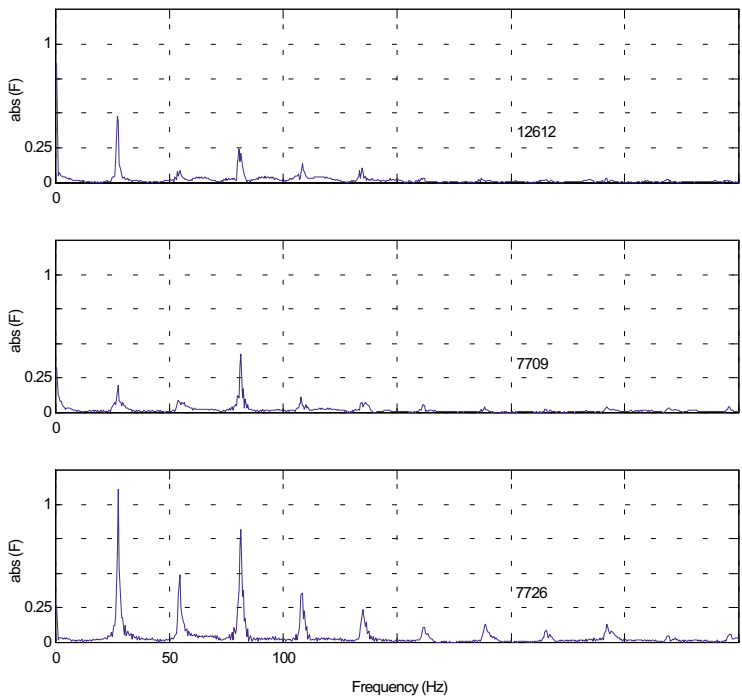

Fig. 5. Magnitude of the Fourier transform of the signals in Fig. 4.

in the system due to the quick deceleration of the model container.

The recorded acceleration time-histories at three different locations within the soil model are shown in Fig. 4. The bottom accelerometer (a7726 in Fig. 1) was positioned near the bottom of the lower sand layer; the middle accelerometer (a7709) near the middle of the container (which is towards the bottom of the upper sand layer); the top accelerometer (a12612) was close to the free surface of compacted sand. Figure 5 shows the results of Fourier analysing these records by the FFT (fast Fourier transform). In each case, the magnitudes of the Fourier coefficients are plotted.

It is clear from these spectra (Fig. 5) that the amplitude of response is generally less in the middle and top locations than at the bottom and that the spectral contents of the signal are different, but no conclusions about the changing response characteristics with time can be deduced from Fig. 5.

\section{Application of time-frequency analysis to these results}

Figures 6, 7 and 8 show time-frequency maps for the three signals in Fig. 4, computed using the harmonic wavelet method. In each case there is a contour plot with a corresponding mesh diagram. Whereas the three graphs in Fig. 4 show the global averages of the frequency responses, Figures 6, 7 and 8 show how the frequency content of these same signals change with time. The soil near the bottom of the model appears to move with the base of the container. This conclusion is drawn from Figure 8 because there is a clearly defined beginning and ending to the recorded acceleration, and the harmonic content of the record remains approximately constant throughout the duration of the excitation. Notice that a sudden start to the excitation acts like an impulse which has a wide frequency composition (theoretically all frequencies from zero to infinity would be present for a delta function input) and that there is a similar, although less pronounced, effect when the excitation suddenly stops.

While the excitation continues, it is apparent that the fundamental frequency of the oscillatory motion is about $27 \mathrm{~Hz}$ and that there are pronounced and continuous harmonics at $54 \mathrm{~Hz}, 81 \mathrm{~Hz}, 108 \mathrm{~Hz}$, and so on. The presence of even as well as odd harmonics is interesting and suggests some loose play or backlash in the driving mechanism such that the pushing half-cycle of the actuator follows a different path from the pulling half-cycle.

The time-frequency maps in Figs 6 and 7 show clearly that the system characteristics are changing with time for the middle and top accelerometers. For the middle accelerometer, Figure 7, the energy at the fundamental frequency, $27 \mathrm{~Hz}$, and in the 2nd harmonic, $54 \mathrm{~Hz}$, diminishes as time passes, but the 3rd harmonic gains energy after an initial lull and higher harmonics tend to grow stronger with time towards the end of the motion.

The same pattern is more exaggerated in Fig. 6, for the top accelerometer. The initial jolt at start-up covers a broad frequency band, and then this accelerometer responds mainly at the fundamental frequency of $27 \mathrm{~Hz}$. The fundamental only begins to diminish after almost a second, as the higher harmonics build up.

Significant changes in soil response may occur at different levels of the model (see Fig. 1). This is because liquefaction effects lead to degradation in the soil's horizontal stiffness. These differences can be explored by plotting differential maps to compare the responses at different levels.

Figure 9 shows the difference between the two maps in Figs 6(A) and 8(A). If Atop is the wavelet array whose absolute magnitude is plotted in Fig. 6(A) and Abot is the corresponding array for Fig. 8(A), abs(Atop)-abs(Abot) is plotted in Fig. 9. Similarly, abs(Amid)-abs(Abot) is plotted in Figure 10 where Amid where Amid is the wavelet array whose magnitude is plotted in Fig. 7(A). 


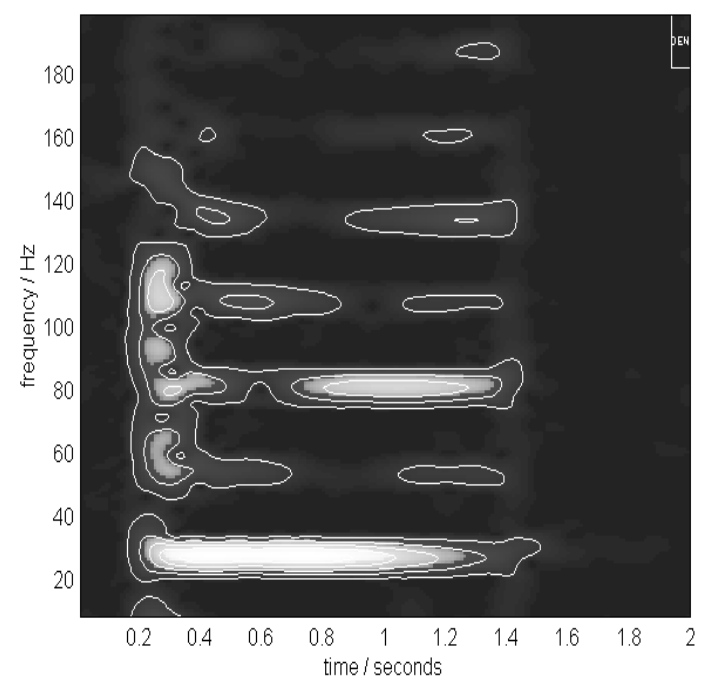

(A)

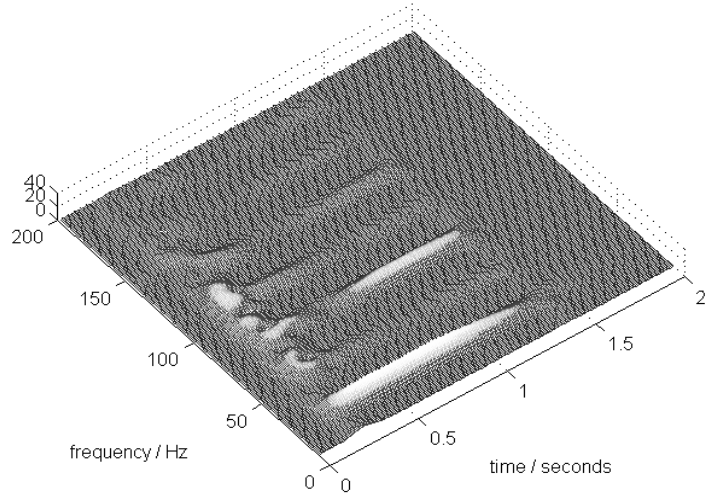

(B)

Fig. 6. (A) Time-frequency map of the changing frequency composition of the top signal, (B) Corresponding mesh diagram for the contour plot in Fig. 6(A).

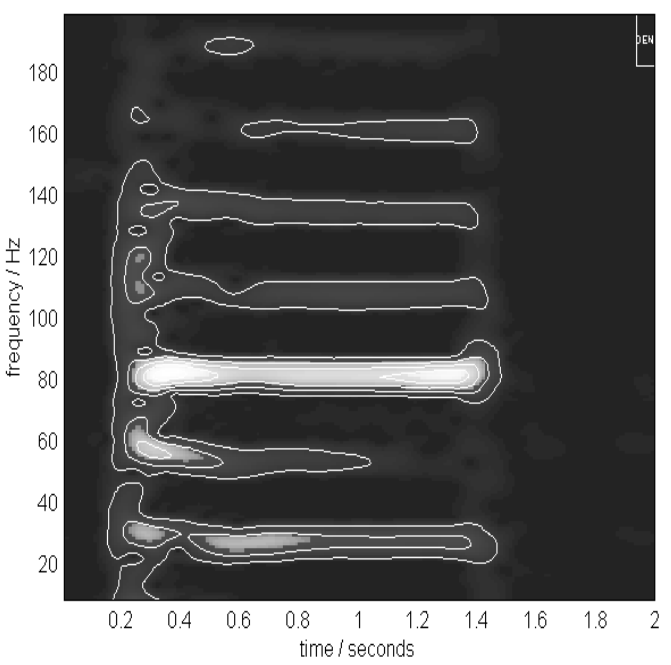

(A)

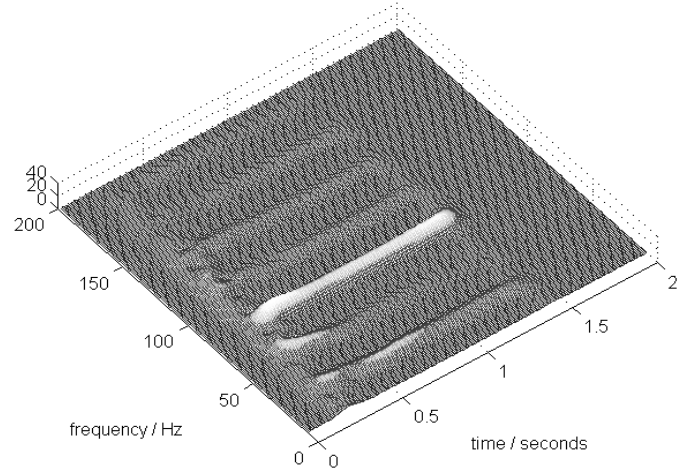

(B)

Fig. 7. (A) Time-frequency map of the changing frequency composition of the middle signal, (B) Corresponding mesh diagram for the contour plot in Fig. 7(A).

\section{Preliminary comments on the observed results}

A simplified explanation of the recorded behavior, as interpreted from the time-frequency maps shown, is as follows.
The dynamic response of each accelerometer depends on the excitation (the time history of the forced motion of the model container) and the frequencyresponse function (FRF) which connects measured output acceleration to excitation. Each FRF depends on the components of the physical system (the type of 


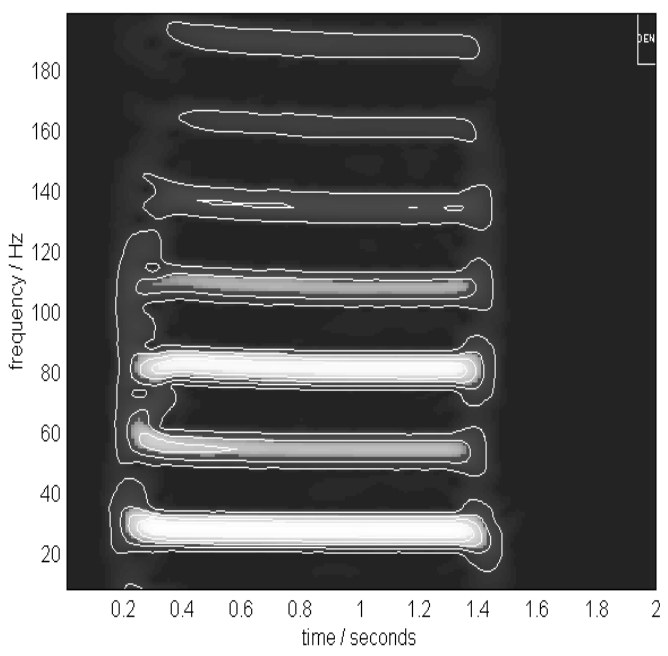

(A)

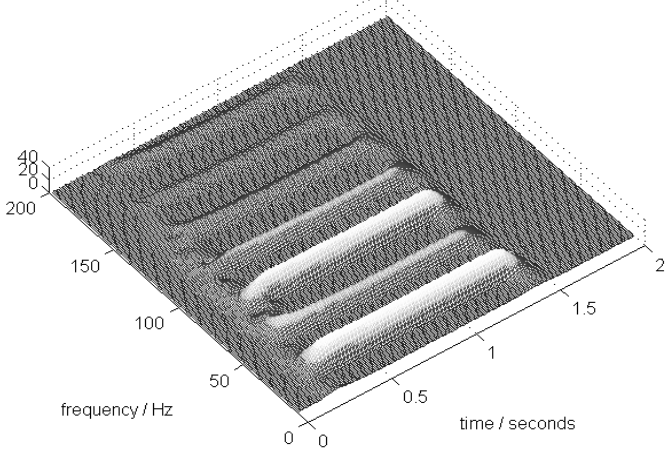

(B)

Fig. 8. (A) Time-frequency map of the changing frequency composition of the bottom signal, (B) Corresponding mesh diagram for the contour plot in Fig. 8(A).

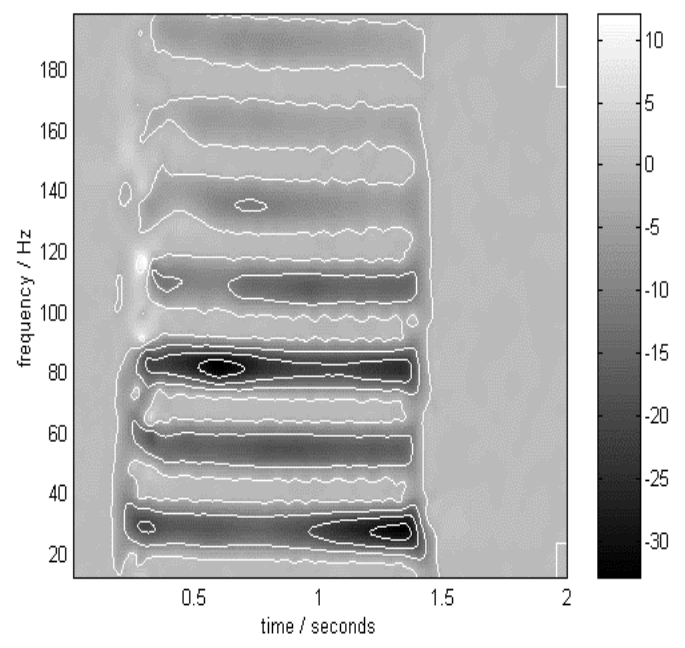

Fig. 9. Differential time-frequency map showing the difference in response measured at the top and bottom of the model i.e. between the maps in Figs 6(A) and 8(A).

sand, how it is arranged, its relative density, moisture content, and position in relation to the walls of its container) and on the amplitude, frequency and duration of excitation. For a linear system, the FRF would depend only on frequency. But in this (nonlinear) application it depends also on amplitude and time.

If the amplitude of each FRF were plotted against frequency it would show resonant peaks where energy

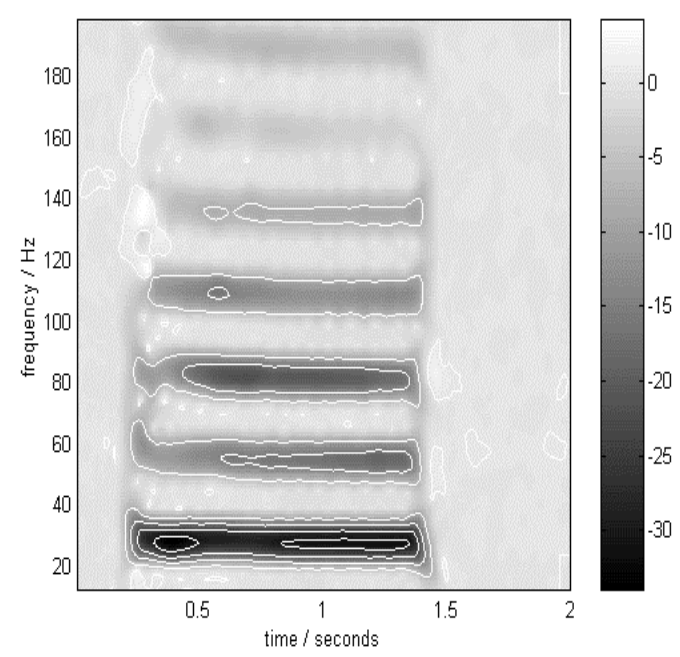

Fig. 10. Differential time-frequency map showing the difference in response measured at the middle and bottom of the model, i.e. between the maps in Figs 7(A) and 8(A).

is transmitted easily, separated by valleys where there is little or no energy transmission. When the excitation has energy in the resonant frequency bands of the FRF, there will be significant response, which shows as a raised peak or ridge on the time-frequency map.

The changing pattern of ridges in Figs 6 and 7, compared with their relatively stable pattern in Fig. 8, suggests that the FRFs that link the response at the top and 
middle of the container with that close to its base are changing with time. Otherwise, the pattern of ridges in Figs 6 and 7, although different from Fig. 8, would be expected not to change with time.

Strictly it is not correct to call an input/output function an FRF if it depends on parameters other than frequency, but the notion is still useful if the effects of changing amplitude and time are slow in relation to the dominant effect of frequency. Similarly, the concepts of natural frequency and resonant response are not strictly true, but they still provide a framework for discussing the measured behavior. Since the bottom accelerometer is close to the floor of its container, to which the excitation mechanism is attached, we consider the output of the bottom accelerometer to define the excitation (approximately).

Figures 6 and 7 demonstrate that the FRF connecting bottom-to-middle and bottom-to-top do change with time. For the middle accelerometer, there is high transmission of the 3rd harmonic (probably due a natural frequency in the vicinity of $81 \mathrm{~Hz}$ ) but reduced transmission at the fundamental frequency of excitation. The 2nd harmonic is similarly attenuated, although higher harmonics remain in play, presumably because of increased wall impacts. For the top accelerometer, there is initially greater response at $27 \mathrm{~Hz}$ and the $3 \mathrm{rd}$ harmonic diminishes before re-establishing itself with greater intensity. For this top accelerometer, there appears to be a significant increase in the higher harmonics with time.

These changes of response function with time are also seen in another way in Figs 9 and 10. These figures show how the response at the top differs from that at the bottom of the model (in Fig. 9) and how the response in the middle of the model differs from that at the bottom (in Fig. 10). If the response functions were all the same, i.e. the model acted as a rigid mass so that all three accelerometers recorded the same signal, then Figures 9 and 10 would display flat surfaces with no hills or valleys. Figure 9 illustrates that the 3rd harmonic is initially lower in magnitude at the top of the model than at its base, with the difference between the fundamental components less, but that this comparison reverses as shaking continues, so that the fundamental at the top becomes significantly less (relatively) than at the bottom, while the 3rd harmonic increases. After the initial shock when excitation is suddenly applied, there is a tendency for all the harmonics at the top and middle of the model (except the third harmonic at the top) to decrease in relative magnitude compared with the bottom of the model because the horizontal stripes tend to darken as motion becomes established after the first shock. From Fig. 10, the fundamental at the middle is relatively lesser than at the base and the higher harmonics at the middle of the box become less significant as time passes.

A full explanation of these characteristics is a challenging problem in physical analysis, but aspects to study in this experiment include the effects of changing the amplitude and duration of the excitation and the soil and container properties. Nonlinear features caused by liquefaction of soil strata, combined, in the model, with end wall effects arising from dynamic incompatibilities between the soil and the container affect the observed dynamic response significantly. These aspects are discussed in the second author's PhD thesis [1] and will be discussed in later papers. Most analysis of earthquakes is in terms of elastic behavior with modifications for nonlinearity of stiffness and damping. The more serious problem is of the irreversible damage in a strong ground motion which cannot be described by nonlinear elasticity theory [4].

We have found that this method of time-frequency analysis provides an important new window for the continuing investigation of these difficult problems.

\section{Acknowledgements}

The experimental work for this analysis was conducted at the US Army Centrifuge Research Center at the Waterways Experiment Station as part of seismic remediation studies of US Army Corps dams under the direction of Dr. M.E. Hynes and Mr. R.H. Ledbetter. The authors acknowledge the support of the US Army Engineer Waterways Experiment Station and the permission of the Chief of Engineers to publish this paper. The authors are also grateful for the assistance of Professors A.N. Schofield of Cambridge University Engineering Department and R.S. Steedman of GIBB Ltd, Reading, UK for their contributions to this paper and research.

\section{References}

[1] Butler, G.D., A Dynamic Analysis of the Stored Angular Momentum Actuator Used with the Equivalent Shear Beam Container, Ph.D. thesis, University of Cambridge, England, 1999.

[2] Cohen, L., Time-Frequency Analysis, Prentice Hall, New Jersey, 1995.

[3] Hodges, C.H., Power, J. and Woodhouse, J., The Use of the Sonogram in Structural Acoustics and an Application to the Vibrations of Cylindrical Shells, J.Sound Vib. 101 (1985), 203218. 
[4] Lee, F.H. and Schofield, A.N., Centrifuge Modelling of Sand Embankments and Islands in Earthquakes, Geotechnique 38(1) (1988), 45-58.

[5] Mark, W.D., Spectral analysis of the convolution and filtering of non-stationary stochastic processes, J. Sound Vib. 11(1970), 9-63.

[6] Newland, D.E., Mechanical Vibration Analysis and Computation, Addison Wesley Longman, 1989.

[7] Newland, D.E., Harmonic Wavelet Analysis, Proc. R.. Soc. Lond. A 443 (1993), 203-225.

[8] Newland, D.E., Random Vibrations, Spectral and Wavelet Analysis, (3rd ed.), Addison Wesley Longman, 1993.

[9] Newland, D.E., Harmonic and Musical Wavelets, Proc. R. Soc. Lond. A 444 (1994), 605-620.

[10] Newland, D.E., Wavelet Analysis of Vibration, Part 1: Theory, J. Vibration \& Acoustics, Trans. ASME 116 (1994), 409-416.

[11] Newland, D.E., Wavelet Analysis of Vibration, Part 2: Wavelet Maps, J. Vibration \& Acoustics, Trans. ASME 116 (1994), 417-425.

[12] Newland, D.E., Wavelet Theory and Applications, Proc. 3rd Int. Congress on Sound and Vibration, Montreal, Canada, 695 713 (Int. Science Publications, AL, USA), 1994. Reprinted as "Wavelet Analysis of Vibration Signals, Part 1", Int. J.
Acoustics and Vib. 1 (1997), 11-16.

[13] Newland, D.E., Time-Frequency Analysis by Harmonic Wavelets and by the Short-Time Fourier transform, Proc. 4th Int. Congress on Sound and Vibration, St. Peters-burg, Russia, 1975-1982 (Int. Science Publications, AL, USA), 1996. Reprinted as "Wavelet Analysis of Vibration Signals, Part 2", Int. J. Acoustics and Vib. 2 (1997), 21-27.

[14] Newland, D.E., Practical Signal Analysis: Do wavelets make any difference?, Proc. 16th ASME Biennial Conf. On Vibration and Noise, Paper DETC97/VIB-4135 (CD ROM ISBN 07918 1243 X), Sacramento, 1997.

[15] Newland, D.E., Application of Harmonic Wavelets to TimeFrequency Mapping, Proc. 5th Int. Congress on Sound and Vibration, 4, 2043-2054 (Paper 260, CD ROM ISBN 876346 06 X), University of Adelaide, Australia, 1997.

[16] Newland D.E., Time-Frequency and Time-Scale Signal Analysis by Harmonic Wavelets, Chapter 1 of Signal Analysis and Prediction, A. Procházka, J. Uhlǐr, P.J.W. Rayner and N.G. Kingsbury, eds., Birkhäuser, Boston, 1998.

[17] Ville, J., Théorie et Applications de la Notion de Signal Analytique, Cables et Transmission 2 (1948), 61-74.

[18] Wigner, E.P., On the Quantum Correction for Thermodynamic Equilibrium, Physical Review 40 (1932), 749-759. 

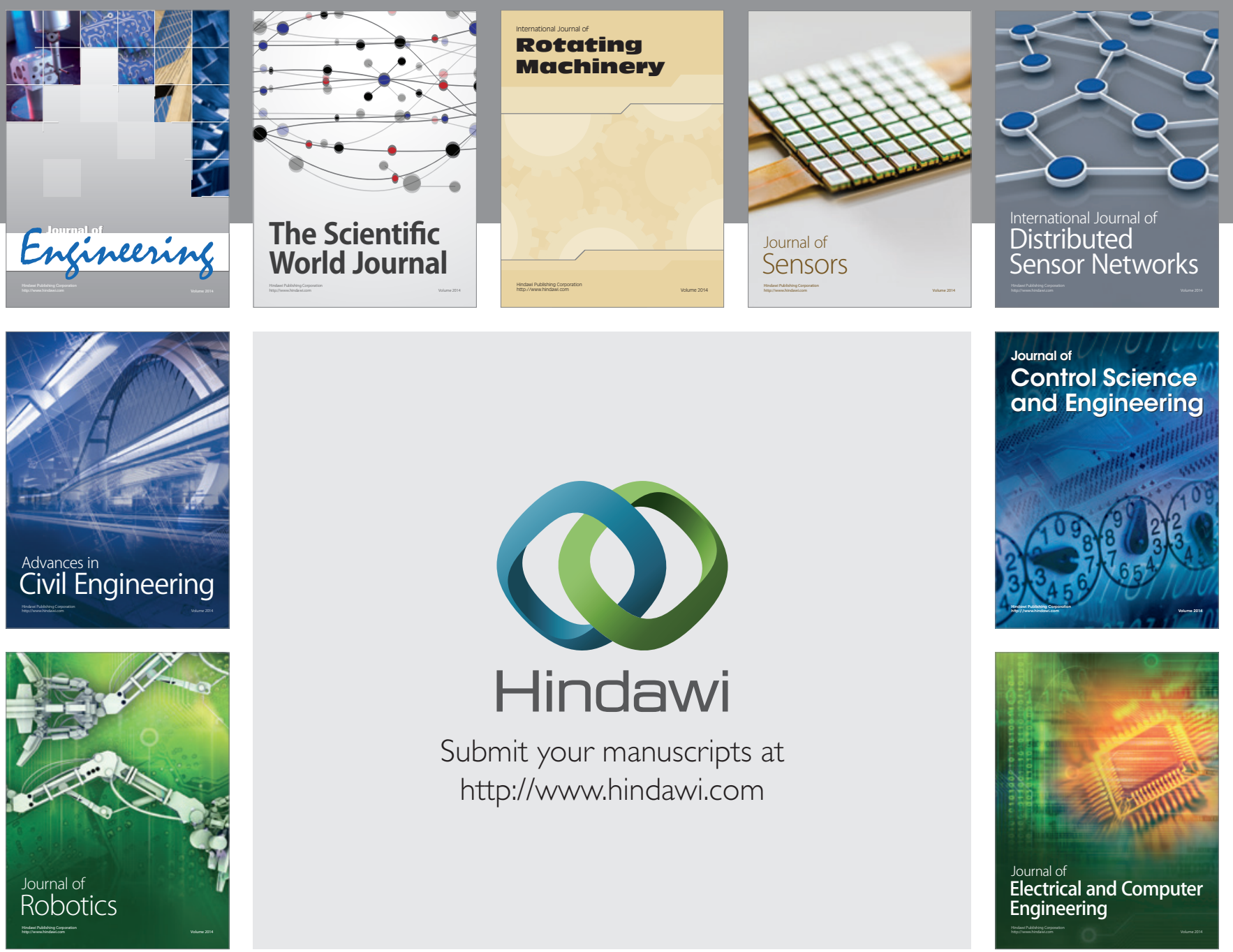

Submit your manuscripts at

http://www.hindawi.com
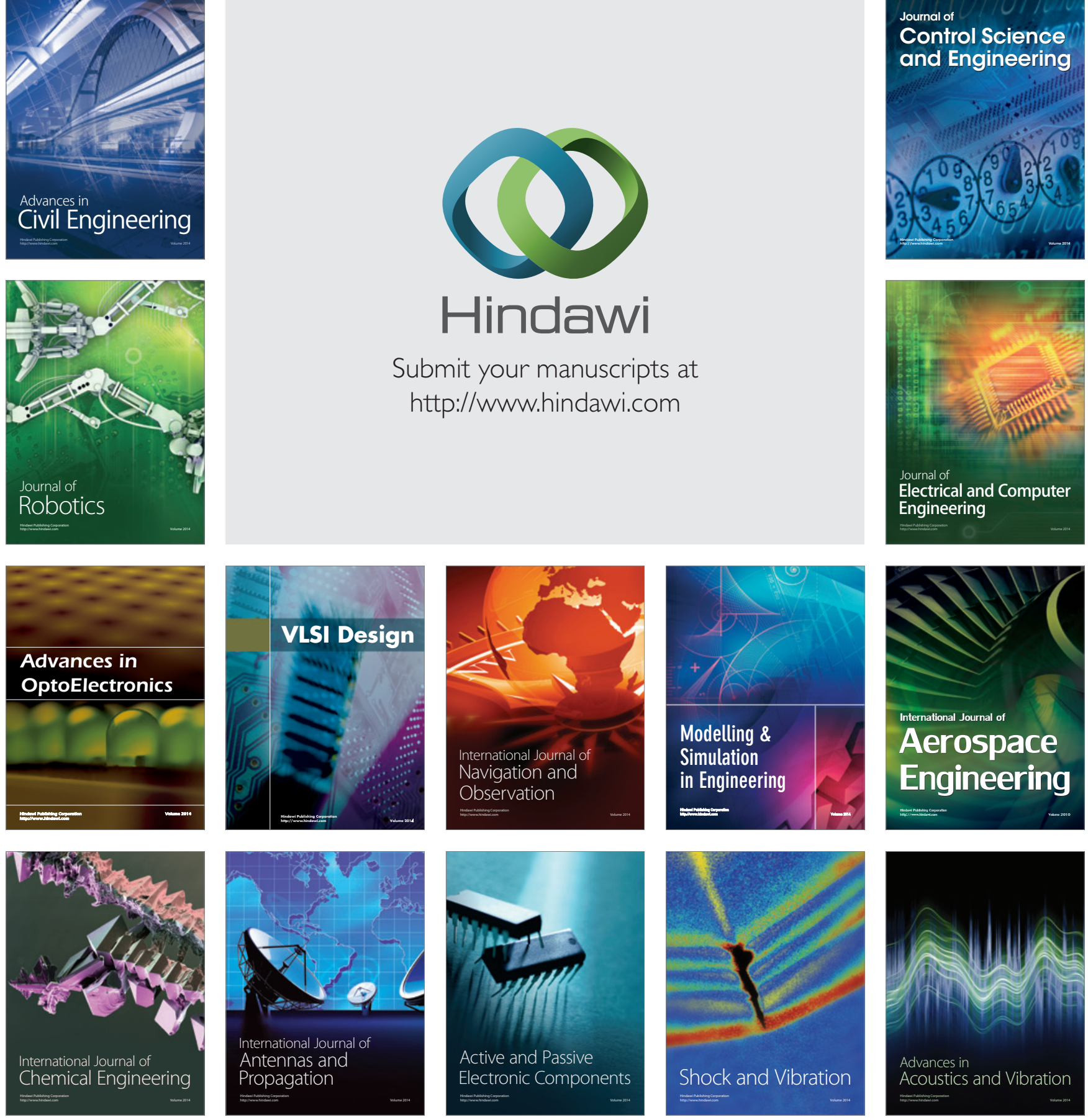